Article

\title{
Optical Characterization of Homogeneous and Heterogeneous Intralipid-Based Samples
}

\author{
Ines Delfino $^{1}$, Maria Lepore ${ }^{2, *}(\mathbb{D})$ and Rosario Esposito ${ }^{3}$ \\ 1 Dipartimento di Scienze Ecologiche e Biologiche, Università degli Studi della Tuscia, 01100 Viterbo, Italy; \\ delfino@unitus.it \\ 2 Dipartimento di Medicina Sperimentale, Università della Campania "L. Vanvitelli", 80100 Napoli, Italy \\ 3 Dipartimento di Ingegneria Chimica, dei Materiali e della Produzione Industriale, \\ Università di Napoli “Federico II”, 80125 Napoli, Italy; rosario.esposito2@unina.it \\ * Correspondence: maria.lepore@unicampania.it
}

Received: 5 August 2020; Accepted: 4 September 2020; Published: 8 September 2020

\begin{abstract}
Different scattering processes take place when photons propagate inside turbid media. Many powerful experimental techniques exploiting these processes have been developed and applied over the years in a large variety of situations from fundamental and applied research to industrial applications. In the present paper, we intend to take advantage of Static Light Scattering (SLS), Dynamic Light Scattering (DLS), and Time-Resolved Transmittance (TRT) for investigating all the different scattering regimes by using scattering suspensions in a very large range of scatterer concentrations. The suspensions were prepared using Intralipid 20\%, a material largely employed in studies of the optical properties of turbid media, with concentrations from $10^{-5} \%$ to $50 \%$. By the analysis of the angular and temporal dependence of the scattered light, a more reliable description of the scattering process occurring in these samples can be obtained. TRT measurements allowed us to obtain information on the reduced scattering coefficient, an important parameter largely used in the description of the optical properties of turbid media. TRT was also employed for the detection of inclusions embedded in Intralipid suspensions, by using a properly designed data analysis. The present study allowed us to better elucidate the dependence of scattering properties of Intralipid suspensions in a very large concentration range and the occurrence of the different scattering processes involved in the propagation of light in turbid media for the first time to our knowledge. In so doing, the complementary contribution of SLS, DLS, and TRT in the characterization of turbid media from an optical and structural point of view is strongly evidenced.
\end{abstract}

Keywords: SLS; DLS; time-resolved transmittance; optical properties; Intralipid

\section{Introduction}

Many physical processes, such as absorption, transmission, fluorescence, and scattering, occur when photons propagate inside turbid media, i.e., media in which the light scattering by medium constituents or generated irregular (randomly distributed) optical nonuniformities are of significant intensity. In particular, when photons are scattered inside these kinds of media, they can undergo different scattering events. If the energy of the scattered photon is the same as the incident photon, the photon-particle interaction is denoted as elastic scattering; if the energy of the scattered photon is lower or higher than the incident photon, the interaction is denoted as quasi-elastic or inelastic scattering. These processes have been widely exploited for the development, over the years, of many experimental techniques that provide a lot of useful information on scattering and structural parameters of the investigated samples, such as size, molecular weight, diffusion coefficient, and hydrodynamic radius [1]. For example, the elastic scattering processes can be analyzed using static light scattering 
(SLS) apparatus [2,3]. Dynamic light scattering (DLS) [2] and diffuse wave spectroscopy (DWS) $[4,5]$ are the experimental techniques related to quasi-elastic scattering processes that have been employed to investigate important theoretical topics and to develop new characterization methods. In particular, DLS can be used in the single scattering regime when photons are scattered only once in the sample. DWS instead works in the multiple scattering regime when photons may be scattered hundreds of times. SLS, DLS, and DWS are usually indicated as light scattering techniques. The inelastic scattering processes are exploited in Raman spectroscopy, which nowadays is one of the most powerful tools in the macro and microscopic biochemical investigations. SLS, DLS, and DWS have been applied in a very large number of research fields spanning from biophysics [6] to microrheology [7,8], microbiology [9], pharmaceutics [10], food characterization [11,12], nanotechnology [13-15] and biomedicine [16,17], just to name a few.

Scattering properties of turbid media can also be investigated by using the time-resolved transmittance (TRT) technique which uses an ultrashort laser source with a pulse duration of few picoseconds or hundreds of femtosecond and a fast detector such as a streak camera or a photon counting system [18-20] to investigate the time behaviour of the light transmitted by the scattering sample. This technique has been largely applied in biomedical research (see [18] and reference therein), [21] but has also offered a certain number of applications in food characterization [22-25] and in technology [26,27].

The above-mentioned techniques represent very powerful tools that are selectively adopted by researchers for studying various samples with different scattering properties in very different experimental conditions as illustrated by the representative cited references and by the enormous number of related papers present in the literature. SLS and DLS require that the samples are highly diluted in order to be in the "single scattering" regime [28,29], while TRT operates in a highly turbid medium or "multiple scattering" regime, and it describes the photon path inside the samples as a diffusive process [30]. In a previous paper [31], we reported a preliminary study on the use of SLS, DLS, and TRT as complementary techniques for the characterization of turbid media from an optical and structural point of view.

In the present paper, we intend to extend the joint use of SLS, DLS, and TRT on a large number of samples that are representative of the different scattering regimes above-mentioned. These samples were prepared using one of the most employed scattering elements used for various applications: Intralipid 20\%. This is an aqueous suspension of lipid droplets suitable for the intravenous feeding of patients; it is largely adopted as a phantom material in biomedical optics due to its low price, very small batch-to-batch variability, and its stability over a long time and at different temperatures [32-34]. By using SLS and DLS, we investigated Intralipid suspensions (here called homogeneous samples) in a large range of concentrations (from $10^{-5} \%$ to $50 \%$ ). They enabled us to scan the different regimes occurring in scattering processes, namely form single to multiple scattering regimes, considering also highly diffusive media [28,29]. To the best of our knowledge, this is the first time that SLS and DLS have been applied to Intralipid samples with such a large range of concentrations. Samples with concentrations varying between $1 \%$ and $50 \%$ were investigated also with TRT. These two series of experiments can be descriptive of all the optical characterization procedures here considered.

In order to further demonstrate the potentialities of scattering-based techniques and properly developed theoretical approaches, we also investigated absorptive inclusions embedded in the above-mentioned suspensions. TRT was employed for studying these samples, whose importance is related to the applications of light propagation in turbid media for imaging reconstruction purposes. These applications are of strategic importance in the development of new approaches in medical imaging.

The plan of the paper is the following. In Section 2, a brief description of light propagation in turbid media is reported, in which the different employed physical parameters and theoretical approaches for the investigated cases are briefly presented. In Section 3, the most relevant information 
about sample preparation and experimental techniques is given. In Section 4 , the experimental results are presented and discussed.

The results here reported show how the use of different techniques allowed us to characterize the optical properties of Intralipid in different scattering regimes confirming the large applicability of SLS, DLS, and TRT in properly chosen experimental conditions. In addition, the experiments performed on inclusions evidenced the potentiality of the developed data analysis procedure in image reconstruction applications.

\section{Light Propagation in Turbid Media}

Light propagation in a turbid medium can be described with a low number of well-defined parameters. To describe the absorption process, a single parameter is needed. This is the absorption coefficient $\left(\mu_{a}\right)$, which has the dimension of the units of reciprocal distance and gives information on the probability of the occurrence of an absorption event. Scattering processes are more complex than absorption to be described, mainly because once a scattering event has occurred, the spatial position of the scattered photon should be provided. Information on the probability of the occurrence of a scattering event is given by the scattering coefficient $\left(\mu_{s}\right)$, which is homologous to $\mu_{a}$. As for the matter of the spatial distribution of the scattered photons, the scattering phase function $p(\theta)$, that is, the relative number of scattered photons as a function of scattering angle, has to be known to fully describe the process. Usually, the full scattering pattern is not known and an anisotropy factor ( $\mathrm{g}$, dimensionless) is given. The proper definition of the anisotropy factor is the following

$$
g=\int_{0}^{\pi} p(\theta) \cos \theta 2 \pi \sin \theta d \theta
$$

The $g$ factor is a measure of the amount of forwarding direction retained after a single scattering event. When a photon is scattered by a particle and its trajectory is deflected by a deflection angle $\theta$, the component of the new trajectory which is aligned in the forward direction behaves like $\cos (\theta)$. There is an average deflection angle, and the mean value of $\cos (\theta)$ is defined as the anisotropy. From the knowledge of the scattering and the anisotropy coefficients, it is possible to define the reduced scattering coefficient, $\mu_{s}{ }^{\prime}=\mu_{s}(1-g)$, which gives information on the probability to have a forward scattered photon. As previously mentioned, the propagation of light in turbid media is dominated by the scattering interaction between a photon and a particle (this term indicating every scattering structure of the sample) which deflects the photon along a new direction of propagation. Scattering techniques are mainly based on signals coming from many scattering structures (particles or nonuniformities) and, thus, provide quantitative measurements of the average structural and dynamical properties of materials. The choice of the scattering technique for analyzing the specific material is mostly set by the characteristic length scales of the structures being probed. Scattering techniques generally work best when the wavelength of the radiation is comparable to the size of the structures that scatter the radiation. For light scattering, the wavelength is about $500 \mathrm{~nm}$, making it ideal for probing soft materials such as tissues, colloids, emulsions, polymer solutions, and surfactant solutions, all of which have structures on length scales from a few hundred to thousands of nanometers. Valuable information on the shape, the size, and the average weight of the scatterers, and on their mutual interactions can be obtained from the SLS technique. Insight into the scatterers' dynamics can be gained by analyzing the fluctuations of the scattered intensity that is the essence of the DLS technique. Transillumination of turbid media with continuum and time-resolved techniques can be used to map the optical parameters, including the reduced scattering and absorption coefficients that characterize the investigated medium, giving average information about the interaction of light with the heterogeneities of the medium itself. 


\subsection{Static and Dynamic Light Scattering}

In the basic scattering geometry adopted for the SLS investigation, the incident laser radiation has a wave vector $\vec{k}_{i}$, pointing in the direction of propagation of the incoming radiation and having an amplitude $\left|\vec{k}_{i}\right|=2 \pi n / \lambda$, where $\mathrm{n}$ is the refractive index of the medium and $\lambda$ is the wavelength of the light in the vacuum. The scattered radiation has a wave vector $\vec{k}_{s}$ that has an amplitude $k_{s}=k_{i}=k$, and points in the direction of the outgoing radiation. The intensity of the scattered light is measured by a detector far from the scattering event by assuming the measurement is performed in the single-scattering limit, i.e., light is scattered no more than one time before exiting the sample. The light scattered from two particles a and $b$ travels towards the detector with a different path length

$$
\Delta \vec{s}=\frac{\overrightarrow{k_{s}}}{k_{s}} \cdot \Delta \vec{r}-\frac{\overrightarrow{k_{i}}}{k_{i}} \cdot \Delta \vec{r}
$$

where $\Delta \vec{r}=\overrightarrow{r_{a}}-\overrightarrow{r_{b}}$ is the relative distance between the two particles. When the size of the scattering volume is much smaller than the distance from the scattering volume to the detector, the phase difference is given by the following equation

$$
\Delta \varphi=k \Delta s=\left(\overrightarrow{k_{s}}-\overrightarrow{k_{i}}\right) \cdot \Delta \vec{r}=\vec{q} \cdot \Delta \vec{r}
$$

where

$$
\begin{gathered}
\vec{q}=\overrightarrow{k_{s}}-\overrightarrow{k_{i}} \\
q=2 k \sin \frac{\theta}{2}
\end{gathered}
$$

The waves scattered from the particles a and $\mathrm{b}$ interfere constructively or destructively according to their relative distance $\Delta \vec{r}$ and to the scattering angle $\theta$, that is the angle between $\vec{k}_{i}$ and $\vec{k}_{s}$. The quantity measured in a scattering experiment is proportional to the scattered intensity $\mathrm{I}(\theta)$ from all the $N$ particles in the scattering volume $V_{s}(q)$ averaged over some periods of time. If the particles are identical spheres, the scattered intensity can be written as:

$$
I(q) \propto|\vec{E}(q)|^{2} S(q)
$$

with

$$
S(q)=\frac{1}{N} \sum_{i, j=1}^{N}<e^{i \vec{q} \cdot\left(\vec{r}_{i}-\vec{r}_{j}\right)}>
$$

where $\vec{E}(q)$ is the average scattered electric field that depends on the scattering properties of a single particle, $S(q)$ is the structure factor that depends only on the relative particle positions $\left(\vec{r}_{i}-\vec{r}_{j}\right)$, and $<>$ denotes the ensemble average. If the quantity $|\vec{E}(q)|$ is known a priori from the experiment or theory, the structure factor $S(q)$ can be retrieved from SLS measurements to investigate the fluctuations in the particle density and the correlation between particles that depends on the relative distance [35]. Equation (6) is too simple for describing the scattering process from nonspherical particles whose size is not negligible. In this case, a more structured expression is considered for describing the angular scattered intensity:

$$
\frac{K C}{R(q)}=\frac{1}{M F(q)}+2 A_{2} C
$$


where

$$
R(q)=\frac{I(q)}{I_{0}} \frac{r^{2}}{V_{s}(q)}
$$

The quantity $R(q)$, called the Rayleigh ratio, is the normalized intensity, and $\mathrm{I}_{0}$ is the intensity of the light beam that illuminates the sample. The quantity $C$ is the weight concentration of the particles with molecular weight $M$. The quantity $F(q)$ is the form factor of the particles, and it is related to the size and shape of particles themselves. $A_{2}$ is the second virial coefficient and measures the nonideality of the solution where the particles are dissolved or the interaction forces between the particles. Different geometrical measurement configurations and theoretical considerations can be adopted to retrieve the molecular weight $M$ and the size of the particles from Equation (8) [36].

The relative distance $\Delta \vec{r}$ between two particles and their phase difference $\Delta \varphi$ change in time, and so the scattered intensity does as it is measured by DLS apparatus. A temporal autocorrelation function can be defined to obtain a quantitative measure of the temporal fluctuations of the scattered light:

$$
\mathrm{g}\left(\mathrm{t}, \mathrm{t}_{0}\right)=\frac{\left\langle I\left(t+t_{0}\right) I(t)\right\rangle}{<I(t)>^{2}}
$$

where $<>$ intends the ensemble average.

The intensity autocorrelation function (Equation (10)) was experimentally measured and was related to another function generally calculated from theory, the field autocorrelation function $e\left(t, t_{0}\right)$, i.e., $\mathrm{e}\left(\mathrm{t}, \mathrm{t}_{0}\right)=\frac{\left\langle E\left(t+t_{0}\right) E(t)\right\rangle}{\langle|E(t)|\rangle^{2}}$, by the relation:

$$
\mathrm{g}\left(\mathrm{t}, \mathrm{t}_{0}\right)=1+\left|\mathrm{e}\left(\mathrm{t}, \mathrm{t}_{0}\right)\right|^{2}
$$

The functional form of $g\left(t, t_{0}\right)$ and its characteristic decay time depends on the dynamics of the scatterers; both were obtained from DLS measurements.

\subsection{Diffusion Approximation}

\subsubsection{Homogeneous Slab}

The light transport through a highly diffusing medium is well described by the diffusion approximation to the radiative transfer equation for the average diffuse intensity [20]:

$$
\left[D \nabla^{2}-\frac{1}{v} \frac{\partial}{\partial t}-\mu_{a}\right] \varphi\left(\vec{r}, t ; \vec{r}_{s}, t_{s}\right)=-\delta\left(\vec{r}-\overrightarrow{r_{s}}\right) \delta\left(t-t_{s}\right)
$$

Here, $v$ is the speed of light in the medium, $\mu_{s}^{\prime}$ is the reduced scattering coefficient, and $\mu_{a}$ is the absorption coefficient. The term $\delta\left(\vec{r}-\overrightarrow{r_{s}}\right) \delta\left(t-t_{s}\right)$ is the point-like source term located at $\overrightarrow{r_{s}}$ at time $t_{s}$, and $D=1 /\left(3 \mu_{s}^{\prime}\right)$ is the photon diffusion coefficient. It should be taken into account that in some cases, $D$ may also depend on the absorption coefficient [19]. Within the extrapolated boundary conditions, the average diffuse intensity $\varphi$ vanishes at extrapolated surfaces outside the turbid medium at a distance $z_{e}=2 \mathrm{AD}$, where the coefficient $\mathrm{A}$ accounts for the refractive index mismatch between the slab and the external medium [37]. Generally, analytical solutions to Equation (12) are only given for simple geometry and homogeneous media, such as the case of the homogeneous slab. The basic solution to this problem for an isotropic source located at a depth $z_{s}=1 / \mu_{s}^{\prime}$ and emitting a light pulse of unit energy at a time $t=0$ is given as:

$$
\varphi\left(\vec{r}, t ; \vec{r}_{s}, t_{s}\right)=\frac{e^{-\mu_{a} v\left(t-t_{s}\right)-\frac{\rho^{2}}{4 D v\left(t-t_{s}\right)}}}{8 \pi^{2} D d_{e}\left(t-t_{s}\right)} \sum_{m=1}^{\infty} e^{-\frac{\pi^{2} m^{2} D v t}{d_{e}^{2}}} \sin \left(\frac{m \pi\left(z_{s}+z_{e}\right)}{d_{e}}\right) \sin \left(\frac{m \pi\left(z+z_{e}\right)}{d_{e}}\right)
$$


where $\rho$ is the radial distance between positions $\vec{r}$ and $\vec{r}_{s}$. The quantities $d_{e}=d+2 z_{e}$ is the extrapolated thickness of the slab.

Taking into account the extrapolated boundary conditions, the outward photon flux measured at the position $\overrightarrow{r_{m}}$ on the surface of the slab is given by $I\left(\vec{r}_{m}, t ; \overrightarrow{r_{s}}, t_{s}\right)=(2 \pi / A) \varphi\left(\overrightarrow{r_{m}}, t ; \vec{r}_{s}, t_{s}\right)$. The quantity I is the reflectance when the source and the detector are upon the same surface and is the transmittance when the source and the detector are upon opposite sides.

In the present case, the quantity of interest for comparing models and experiments in TRT was the transmitted light in a specific position as a function of time. According to Contini et al. [38], the expression describing the transmitted light, $T(t)$, is obtained as a solution of the diffusion equation (Equation (12)) considering point-like sources and extrapolated boundary conditions

$$
T(t)=\frac{\exp \left(-\mu_{a} v t\right)}{4 \pi D A(n) d_{e} t} \sum_{n=1}^{\infty} \exp \left(-\frac{D v t \pi^{2} n^{2}}{d_{e}^{2}}\right) \sin \left(\frac{n \pi\left(z_{s}+z_{e}\right)}{d_{e}}\right) \sin \left(\frac{n \pi\left(d+z_{e}\right)}{d_{e}}\right)
$$

\subsubsection{Heterogeneous Slab}

In order to describe the effects of the presence of an inclusion hidden in an otherwise homogenous slab (Figure 1), a perturbation approach to the diffusion equation was needed. We make the assumption that the difference between the optical coefficient of the inclusion and the host media is radially described by a Gaussian law, as expressed by the form.

$$
\mu(\rho)=\Delta \mu \exp \left(-\ln 2\left(\frac{\rho}{R}\right)^{2}\right)
$$

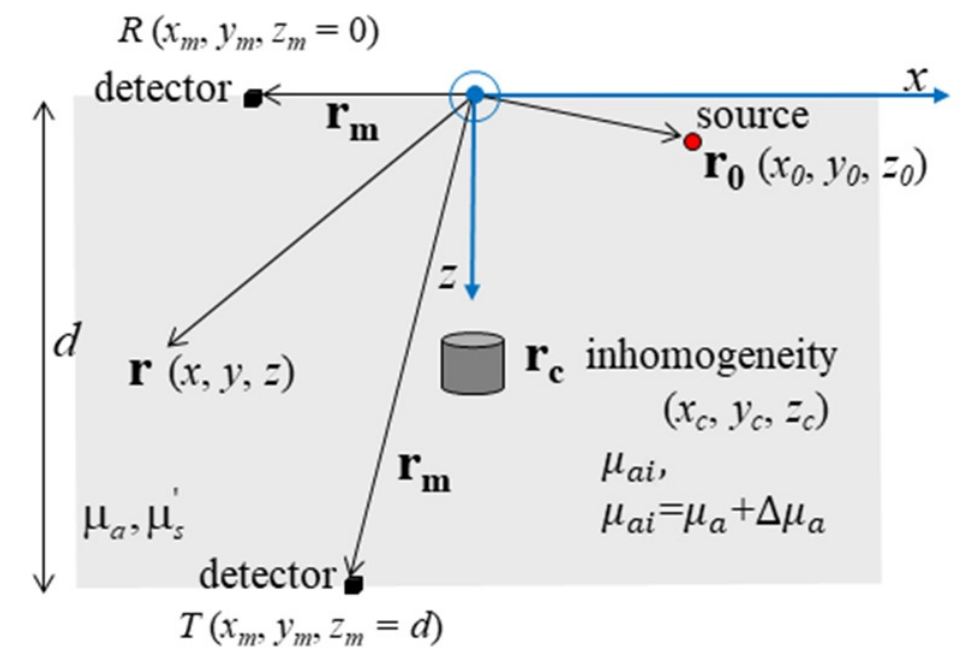

Figure 1. Schematic arrangement of a heterogeneous slab with an absorptive inclusion.

The quantity $\Delta \mu$ is the perturbation intensity, and it is observed at the centre of the inclusion. The quantity $R$ is defined as the radius of the inclusion and corresponds to the width at half maximum of the Gaussian curve. It can be shown that at first-order perturbation approximation, the outward photon flux I transmitted in presence of a defect can be written as the sum of three terms, as follows

$$
I_{\text {pert }}\left(t ; z_{\text {incl }}\right)=I(t)+\delta I_{\mu_{a}}\left(t ; z_{\text {incl }}\right)+\delta I_{\mu_{s}^{\prime}}\left(t ; z_{\text {incl }}\right)
$$

The first term describes the photon flux transmitted through the homogeneous slab. The second and third term, the quantities $\delta I$, describe, at first approximation, the change in the outward photon 
flux due to the presence of an absorptive and a scattering inclusion, respectively. Here, we present the analytical forms obtained for a Gaussian absorptive and scattering inclusion:

$$
\begin{aligned}
& \delta I_{\mu_{a}}\left(t ; z_{\text {incl }}\right)=\frac{\Delta \mu_{a} \exp \left(-\mu_{a} v t\right)}{2 \pi^{2} A(n) D^{2} d_{e}^{2} t} \sum_{k, l=1}^{\infty} R_{k, l}(R, t) \cdot Z_{k, l}\left(h, z_{\text {incl }}, t\right) \\
& \delta I_{\mu_{s}^{\prime}}\left(t ; z_{\text {incl }}\right)=\frac{\Delta \mu_{s}^{\prime} \exp \left(-\mu_{a} v t\right)}{8 \pi^{2} d_{e}^{4} A(n) t} \sum_{k, l=1}^{\infty} R_{k, l}^{\prime}(R, t) \cdot Z_{k, l}^{\prime}\left(h, z_{\text {incl }}, t\right)
\end{aligned}
$$

In both cases, a double series characterized by a factor and the product of two functions is present. The former is a function of the radius of the inclusion, the latter depends on inclusion longitudinal extension. So, the parameters that characterize the perturbation induced by the inclusion are its radius, height, and the perturbation intensity value $\Delta \mu_{a}$ and $\Delta \mu_{s}^{\prime}$.

The accuracy of the proposed perturbation model can be investigated by considering the time-resolved contrast function, that is defined as follows

$$
C\left(t, z_{p c}, \Delta \mu\right)=\frac{I_{p e r t}\left(t, z_{p c}, \Delta \mu\right)-I(t)}{I(t)}
$$

So, the contrast function is the relative change in the outward photon flux due to the presence of the inclusion. As defined, the contrast depends on the size of the inclusion and its perturbation intensity value.

In order to investigate the accuracy of the Gaussian perturbation model, we numerically solved the diffusion equation by using a Finite Element Method (FEM) for a slab with a thickness of $d=40 \mathrm{~mm}$, absorption coefficient $\mu_{a}=0.01 \mathrm{~mm}^{-1}$, reduced scattering coefficient $\mu_{s}^{\prime}=1.0 \mathrm{~mm}^{-1}$, assuming that the inclusion had a diameter equal to the height. In Figure 2a, the absorptive contrast (Equation (19)) measured in transmittance configuration as a function of time for an absorptive inclusion of radius $R=5 \mathrm{~mm}$ and $\Delta \mu_{a} / \mu_{a}=0.2$ located at different depths inside the slab is shown. The solid curves are the FEM simulations, i.e., the exact solution, the dashed curves are the perturbation model predictions. The contrast function increased as the inclusion moved toward the boundary surfaces.

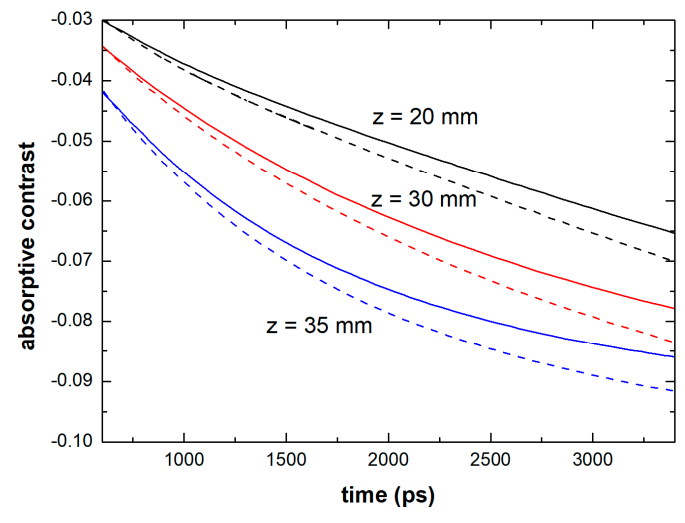

(a)

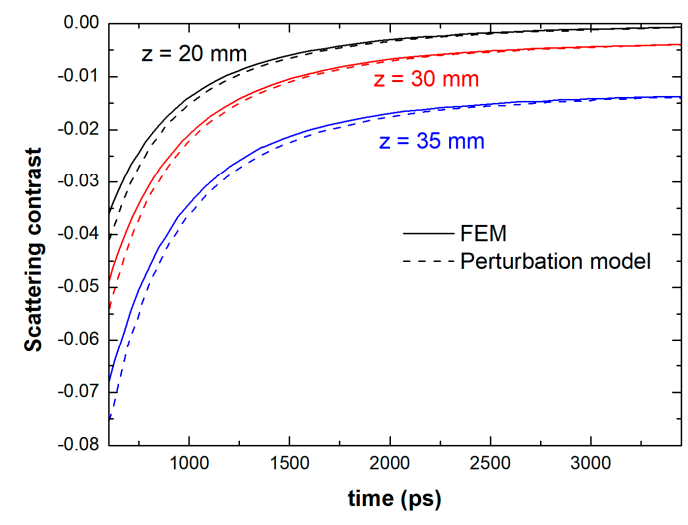

(b)

Figure 2. (a) The absorptive contrast of an inclusion with $\Delta \mu_{a} / \mu_{a}=0.2$ located at different depth inside a slab with a thickness of $d=40 \mathrm{~mm}$, reduced scattering coefficient $\mu_{s}{ }^{\prime}=1.0 \mathrm{~mm}^{-1}$, absorption coefficient $\mu_{a}=0.01 \mathrm{~mm}^{-1}$. (b) The scattering contrast of an inclusion with $\Delta \mu_{s}{ }^{\prime} / \mu_{s}{ }^{\prime}=0.2$ located at different depth inside a slab. The optical properties of the slab are the same as (a).

For each considered depth of the inclusion, the contrast increased as time increased. The accuracy of perturbation model prediction was better than $7 \%$ for a relative perturbation intensity of less than $20 \%$. In Figure $2 \mathrm{~b}$, the scattering contrast (Equation (19)) in transmittance measurement configuration 
as a function of time for a purely diffusive inclusion located at the same depth of the absorptive inclusion is shown. Conversely to the case of an absorber, the diffusive contrast increased as the time decreased toward to the ballistic time. As before, the diffusive contrast increased also as the inclusion moved close to the surfaces of the slab. A good agreement between FEM simulations and approximated solutions was observed, and, furthermore, the different temporal behaviour of the absorptive and diffusive contrast allowed one to discriminate between the optical properties of the inclusion.

The accuracy of the first-order approximation method is limited to weak perturbations of a few percent. To overcome this limitation, a closed-form expression for the average diffuse intensity (Equation (12)) in the case of a steady-state diffusion equation for a turbid slab in the presence of an absorbing defect was derived through an iterative approximation scheme [39]:

$$
\Delta \varphi\left(\vec{r}, \overrightarrow{r_{0}} ; \Delta \mu_{a}\right)=\left(-\Delta \mu_{a} V+\frac{\Delta \mu_{a}^{2} V^{2} \bar{\varphi}\left(\vec{r}_{c}\right)}{1+\Delta \mu_{a} V \bar{\varphi}\left(\overrightarrow{r_{c}}\right)}\right) \frac{\varphi\left(\vec{r}_{c}-\overrightarrow{r_{o}}\right) \bar{\varphi}(\vec{r})}{4 \pi}
$$

where $\Delta \phi$ is the perturbation of the average intensity, and $\varphi$ is the solution of the steady-state diffusion equation for the homogeneous slab and a point-like source located at $\vec{r}_{0}$ :

$$
\varphi\left(\vec{r}-\overrightarrow{r_{o}}\right)=\frac{1}{4 \pi D} \sum_{m=-\infty}^{\infty} \frac{\exp \left(-\sqrt{\frac{\mu_{a}}{D}} \rho_{+}\right)}{\rho_{+}}-\frac{\exp \left(-\sqrt{\frac{\mu_{a}}{D}} \rho_{-}\right)}{\rho_{-}}
$$

with the quantities

$$
\rho_{ \pm}^{2}=\rho^{2}+\left(z-z_{ \pm m}\right)
$$

The square of the radial distance from the z-axis is $\rho^{2}=\left(x-x_{0}\right)^{2}+\left(y-y_{0}\right)^{2}$. The quantities $z_{+m}$ and $z_{-m}$, given by

$$
\begin{gathered}
z_{+m}=2 m d_{e}+z_{s} \\
z_{+m}=2 m d_{e}-2 z_{e}-z_{s}
\end{gathered}
$$

define the locations of the positive and negative point-like sources along the z-axis. The quantity $\bar{\varphi}$ is its average value over the volume $\mathrm{V}$ of the inclusion (Figure 1):

$$
\bar{\varphi}(\vec{r})=\frac{1}{V} \iiint_{V} d^{3} \vec{r}^{\prime} \varphi\left(\vec{r}-\vec{r}^{\prime}\right)
$$

Figure 3 shows the relative contrast in the case of a black defect $\left(\Delta \mu_{a}>\infty\right)$ versus the inclusion radius: the red dotted curve is the model prediction as given by Equations (20) and (21), and the solid black curve is the result of the Monte Carlo (MC) simulation [39]. The results are obtained for an inclusion located midway between the source and the detector at $z_{\mathcal{c}}=\mathrm{d} / 2$ in a coaxial configuration. The host medium had a reduced scattering coefficient of $\mu_{s}{ }^{\prime}=1.0 \mathrm{~mm}^{-1}$, absorption coefficient of $\mu_{a}=0.01 \mathrm{~mm}^{-1}$, and thickness of $d=40 \mathrm{~mm}$. As can be noticed, the theoretical predictions are in good agreement with MC simulations for the radius of the black defect ranging from 2 to $5 \mathrm{~mm}$. In particular, an excellent agreement between the theory and the MC results was obtained for the radius lower than $4 \mathrm{~mm}$. Indeed, the discrepancies were lower than $3 \%$ for the radius of the inclusion increasing from 2 to $4 \mathrm{~mm}$. 


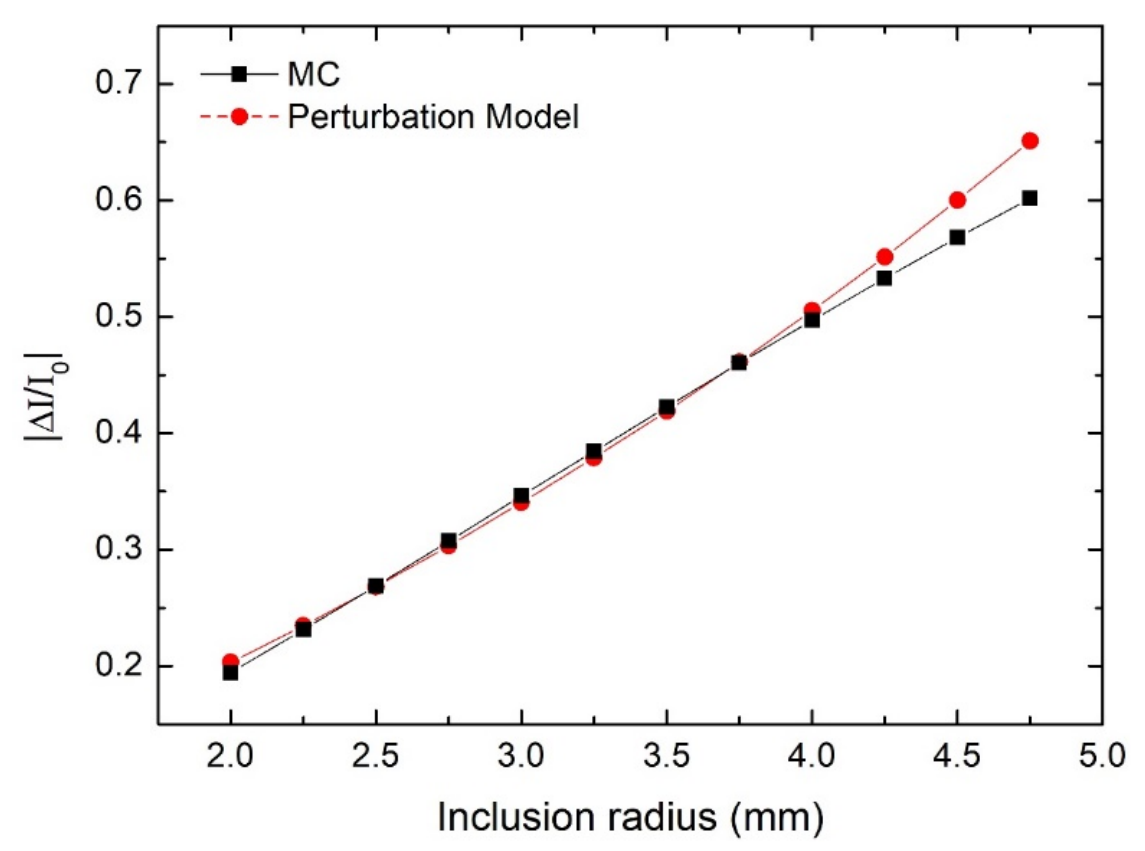

Figure 3. Relative contrast as a function of the inclusion radius of the black defect. The red curve is the perturbation model prediction and the black curve is the MC simulation.

\section{Materials and Methods}

\subsection{Sample Preparation}

Intralipid ${ }^{\circledR}$ is a sterile aqueous suspension of lipid droplets usually employed for intravenous feeding of patients. It is offered as Intralipid-10\%, Intralipid-20\%, and Intralipid-30\% (the percentage signifies the relative amount of lipids: $10 \%$ indicates $10 \mathrm{~g}$ of lipid per $100 \mathrm{~mL}$ of suspension). According to the manufacturers, a $500 \mathrm{~mL}(497.75 \mathrm{~g})$ bottle of Intralipid- $10 \%$ consists of soybean oil $(50 \mathrm{~g})$, lecithin $(6 \mathrm{~g})$, and glycerin $(11.25 \mathrm{~g})$ in water $(430.5 \mathrm{~g})$. The resulting suspension is mainly composed of quasi-spherical colloidal particles with a size in the $25-625 \mathrm{~nm}$ range, consisting of soybean oil encapsulated within a $2.5-5 \mathrm{~nm}$-thick monolayer membrane of lecithin [40]. The glycerin is dispersed into individual molecules in water. Accordingly, the suspension is usually described as a suspension of concentric spheres. In Intralipid-10\%, approximately one half of the amount of available lecithin constitutes the membrane encapsulating the soybean oil, and one half forms small bilayer vesicles. Intralipid 20\% was used for preparing liquid suspensions and absorbing cylindrical inclusions used in the present investigation.

\subsubsection{Liquid Suspensions}

Aqueous suspensions of Intralipid 20\% (Pharmacia, Rockville, MD, USA) in distilled water at different values of the volume concentration, $\mathrm{C}$, ranging from $10^{-5} \%$ to $50 \%$ were prepared (thirty-five different concentrations) and investigated with the cited experimental methods. Moreover, aqueous suspensions of a fixed concentration of Intralipid (3.5\%) and different controlled quantities of black India ink were also prepared for TRT measurements aimed at quantifying the absorption coefficient. In particular, twenty different ink concentrations from $0 \%$ to $0.6 \%$ were considered. For each concentration, three samples were prepared and investigated. All the suspensions were prepared by using a magnetic stirrer.

\subsubsection{Absorptive Cylindrical Inclusions}

In order to test the validity of the approach proposed in Section 2.2.1 Paragraph, absorptive cylindrical inhomogeneities with a thickness $h$ equal to their diameter $2 R(h=2 R=15 \mathrm{~mm})$ were 
prepared using a fast and easily implemented procedure [41]. A highly purified agarose powder was dissolved in distilled water at $1 \%$ concentration and heated up to the melting temperature $\left(95^{\circ} \mathrm{C}\right)$. The agarose solution alone has negligible absorption and very low turbidity. Thus, the desired optical properties of the phantom were obtained by adding appropriate amounts of Intralipid as a scattering medium, and black India ink (Rotring) as an absorbing medium, taking into account that agar induced a systematic decrease in the $\mu_{s}{ }^{\prime}$ value. The absorption and the reduced scattering coefficients of the solid slabs were varied by changing the concentrations of both the black ink and the Intralipid and were determined as previously described for the liquid host medium. Solid cylindrical inclusions with a reduced scattering coefficient $\mu_{s}{ }^{\prime}=0.520 \mathrm{~mm}^{-1}$ and with different absorption coefficients (namely, $\mu_{a}=0.0069 \mathrm{~mm}^{-1}, 0.0077 \mathrm{~mm}^{-1}, 0.0089 \mathrm{~mm}^{-1}, 0.0095 \mathrm{~mm}^{-1}, 0.010 \mathrm{~mm}^{-1}, 0.011 \mathrm{~mm}^{-1}$ ) were used. These values correspond to relative changes in the absorption coefficient of the phantom $\left(\mu_{a, \text { incl }}-\mu_{a}\right) / \mu_{a}=\Delta \mu_{a} / \mu_{a}$ equal to $46 \%, 64 \%, 88.3 \%, 102 \%, 118 \%$, and $136 \%$, respectively.

\subsection{Experimental Techniques and Data Analysis Procedures}

\subsubsection{Static and Dynamic Light Scattering Experiments}

In static light scattering, the time-averaged-intensity of the laser light scattered by the medium is measured at various fixed angles. Rayleigh's theory allows the prediction of the angular intensity distribution of the light scattered by particles with a radius $(R)$ resulting to be much smaller than the wavelength of the impinging light $(\mathrm{R}<\lambda / 20)$. The employed experimental setup is reported in Figure 4. Tens of milliwatts from a doubled Nd:YAG laser $(\lambda=532 \mathrm{~nm}$, Excel, Laser Quantum, maximum power $1.8 \mathrm{~W}$ ) were used. A $130 \mathrm{~mm}$-focal length lens was employed to focalize the beam on a cylindrically-shaped $10 \mathrm{~mm}$-diameter glass cuvette, containing the sample.

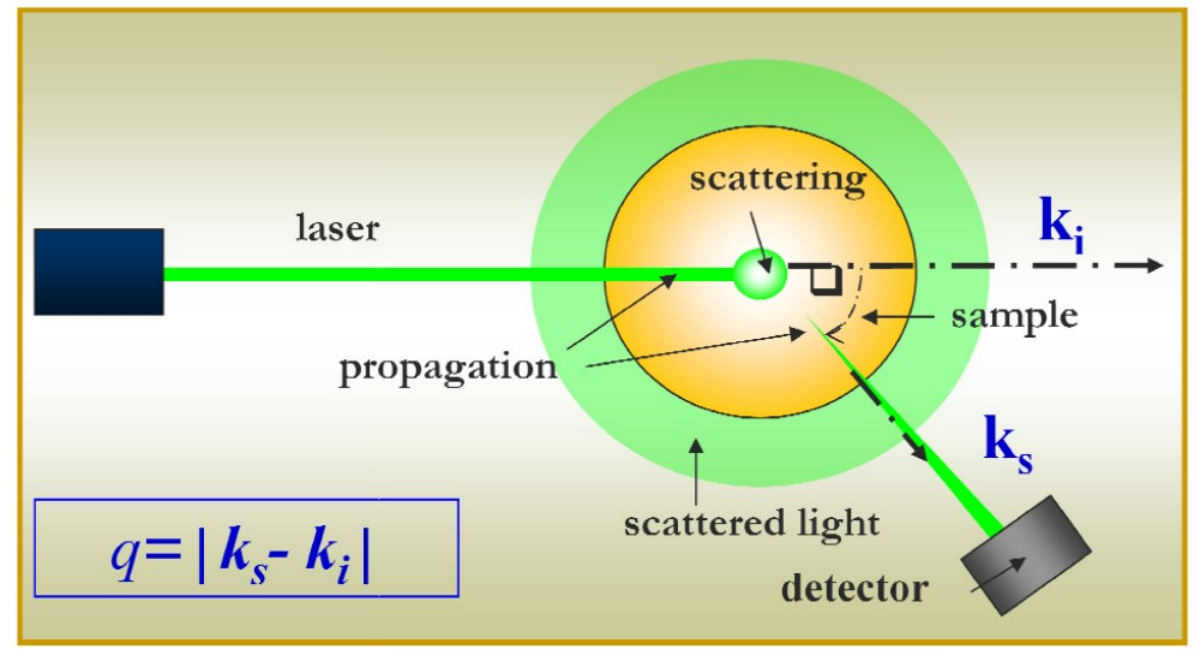

Figure 4. Static and dynamic light scattering experimental set-up.

The light coming from the sample-containing cuvette was collected by an optical system and sent to a photomultiplier (Hamamatsu HC120, Hamamatsu, Shizuoka, Japan). Both the optical system and the photodetector were mounted onto a motorized goniometric rotating device. Rotating the system, the light intensity at different angles could be detected in the range between 15 and $155^{\circ}$, with a maximum resolution of $0.1^{\circ}$. The system worked in a photon-counting detection mode. As previously stated, the angular dependency of the scattered intensity depends on the characteristics of scattering inhomogeneities inside the analyzed sample. Because of the limits in the angular detection range, the intensity scattered at angles close to $180^{\circ}$ had to be eventually extrapolated, using proper interpolation procedure. The data were analyzed by means of the available Brookhaven 
SLS software (Brookhaven Instruments, Holtsville, NY, USA). The same apparatus was also used for DLS measurements.

The DLS technique often investigates the fluctuations of the temporal light intensity scattered in a well-defined volume of the sample (called scattering volume) [2]. The dynamics of the particles in a solution are mainly responsible for the intensity fluctuations of the light scattered by those particles. When the Brownian motion is dominating, the average diffusion coefficient of the scatterers can be retrieved by analyzing the scattered light intensity autocorrelation function (ICF). This latter is obtained by performing the temporal correlation of the scattered light intensity with itself. The normalized ICF for the monomodal size distribution of spherical particles, diffusing in a homogeneous liquid, can be written as

$$
g(t)-1=\exp (-2 \Gamma t)
$$

where

$$
\Gamma=\frac{1}{\tau}=D_{b r} \mathrm{q}^{2}
$$

and $\tau$ is the time characteristic of the process and $q$ is the scattering vector defined in Equation (5). $D_{b r}$ is the diffusion coefficient that can be used to determine the hydrodynamic radius of the diffusing particle, $R_{h}$, that is defined as the radius of a hypothetical sphere that diffuses at the same rate as a particle under investigation. The relation between diffusion coefficient and the hydrodynamic radius is given by the Stokes-Einstein equation:

$$
D_{b r}=\frac{k_{B} T}{6 \pi \eta R_{h}}
$$

where $k_{B}$ is the Boltzmann coefficient $\left(1.380 \times 10^{-23} \mathrm{~kg} \mathrm{~m}^{2} \mathrm{~s}^{-2} \mathrm{~K}^{-1}\right)$, $\mathrm{T}$ is an absolute temperature, and $\eta$ is the medium. The translational frictional coefficient, $f$ (i.e., frictional force suffered by particles experiencing the Brownian motion divided by the velocity of the particle) can be obtained using the following equation [42]

$$
f=\frac{\mathcal{R} T}{N_{A} D_{b r}}
$$

where $\mathcal{R}$ is the gas constant $\left(8.314 \times 10^{-7} \mathrm{erg} \mathrm{mol}^{-1} \mathrm{~K}^{-1}\right)$. The translational frictional coefficient offers information on the shape of diffusing macromolecules.

For DLS measurements, the scattered light was detected at a right angle $\left(\theta=90^{\circ}\right)$ scattering geometry by a photomultiplier. The detected signal is then passed to a BI-9000 (Brookhaven Instruments, Holtsville, NY, USA) digital correlator. Data analysis was performed by using BI9000 software (Brookhaven Instruments, Holtsville, NY, USA) [2].

\subsubsection{Time-Resolved Transmittance Experiments}

An experimental apparatus used for time-resolved measurements of Intralipid suspensions and inclusions is generally composed by an ultrashort laser source as a Ti: Sa laser that is able to give pulses with a time duration of hundreds of femtoseconds or pulsed diode laser with a pulse duration of tens of picosecond. In both cases, near-infrared radiation is used, i.e., with wavelengths around $800 \mathrm{~nm}$. The detectors are streak cameras or time-correlated single-photon counting (TSPC) systems. Streak cameras offer a time resolution of a few picoseconds, while TSPC systems usually ensure a time resolution of tens of picoseconds. Detailed descriptions of similar experimental set-ups are reported in $[19,20,43,44]$.

For measurements on liquid suspensions, cuvettes of various thicknesses were used to satisfy all the assumptions of the diffusion approximation even for Intralipid low concentrations. For all the examined samples, the cuvette size prevented boundary-reflection problems.

By means of the TRT technique, the optical properties (namely, the absorption coefficient, $\mu_{a}$, and the reduced scattering coefficient, $\mu_{s}{ }^{\prime}$ ) of the homogeneous Intralipid suspensions were obtained, 
by using the analytical expression for the transmitted light reported in Equation (14). The expression was rewritten to introduce a set of three fitting parameters: RA0, an amplitude factor; $\mu_{a}$; and $\mu_{s}{ }^{\prime}$, forming the parameter vector $\alpha=\left(\mathrm{RA} 0, \mu_{a}, \mu_{s}{ }^{\prime}\right)$ [19,44]. After subtraction of the background noise, the experimental data were fitted to the theoretical prediction by minimization of the $\chi^{2}$ parameter. The minimization procedure was carried out by the use of a home-made code employing Migrad, and Nelder and Mead minimization procedures. If these methods failed to find a real minimum, a Monte Carlo minimization procedure was also employed.

It is worth to note that TRT experiments allow the determination of $\mu_{s}{ }^{\prime}$ and $\mu_{a}$ that can be related to the photon diffusion constant D. It is interesting to note that the photon diffusion coefficient $\mathrm{D}$ that is present in Equation (14) that describes the photon propagation inside turbid media can be related to another interesting scattering property named the transport mean free path, $\ell^{*}$. The mean free path represents the distance travelled by photons between two subsequent scattering events [45]. In particular, the following relationship can be considered: $\mathrm{D}=(1 / 3) \mathrm{v}_{\mathrm{e}} \ell^{*}$, where $\mathrm{v}_{\mathrm{e}}$ is the transport velocity.

For measurements on absorptive cylindrical inclusions placed inside the host medium, a rectangular cell with an internal thickness of $40 \mathrm{~mm}$, a height of $145 \mathrm{~mm}$, and a width of $130 \mathrm{~mm}$ was adopted. The cell was fabricated using black polyvinyl chloride with two small transparent apertures to reduce inner boundary reflections. Each cylindrical inclusion was placed in the center of the cell by means of a nylon wire. The photon source, the cylinder axis and the detector were kept aligned within $0.5 \mathrm{~mm}$. The time-resolved transmittance data were accumulated at intervals of $5 \mathrm{ps}$. At the beginning of data processing, the center of each reference pulse was determined [19], and the temporal axis of each profile was arranged so that the time at which the beam hit the phantom surface was assumed equal to zero. The errors related to the identification of pulse centers can cause noise in the final data. Point measurements were carried on by translating the inclusion inside the host medium along the $\mathrm{x}$-direction from $\mathrm{x}=-40 \mathrm{~mm}$ to $\mathrm{x}=40 \mathrm{~mm}$ with a $1 \mathrm{~mm}$ step, transversally to the source-detector axis. The obtained profiles described the temporal distributions of the transmitted light through the phantom and were employed to determine the absorption coefficient of the inclusions.

For each inclusion, the Gaussian perturbation model was used for performing the fit with the experimental profiles for each scanning position. The perturbation intensity was the only parameter of the fitting.

\section{Results and Discussion}

\subsection{SLS Results}

The angular dependence of the scattered intensity (corrected for the volume effect, i.e., the quantity $\operatorname{Isin} \theta$ ) is reported as measured by the SLS apparatus for Intralipid solutions at various concentrations in Figure 5a. In particular, to get rid of the absolute intensity, the relative scattered intensity (i.e., $\left.\mathrm{I}_{\theta} \sin \theta /\left(\mathrm{I}_{90} \sin \left(90^{\circ}\right)\right)\right)$ was considered. For the low scattering samples (those with an Intralipid concentration in the order of $0.16 \%$ or lower), the highest intensity was detected at small angles, that is, light scattered in the forward direction greatly exceeded light scattered in the other directions. For highly scattering samples (namely, samples with Intralipid at volume concentrations of $10 \%$ or higher), the light intensity detected in conditions near to the backscattering exceeded that of the light scattered in the forward direction. It is known that for solutions with intermediate values of the Intralipid concentration (around $0.16 \%<\mathrm{C}<3 \%$ ), the collected intensity is less dependent on the detection angle even if the intensity detected at great angles is higher than that recorded near to the forward scattering conditions. This was witnessed by data reported in Figure 5b where SLS resulted in $0.7 \%, 0.875 \%, 1.4 \%$, and $2.175 \%$ - concentration of Itralipid suspensions obtained by Feder et al. by using a $633 \mathrm{~nm}$ laser light is reported. Despite the different radiation wavelength, the qualitative behavior can be compared to the data reported here, since the ratio between impinging radiation wavelength and average scatterer size [40] is quite similar. 


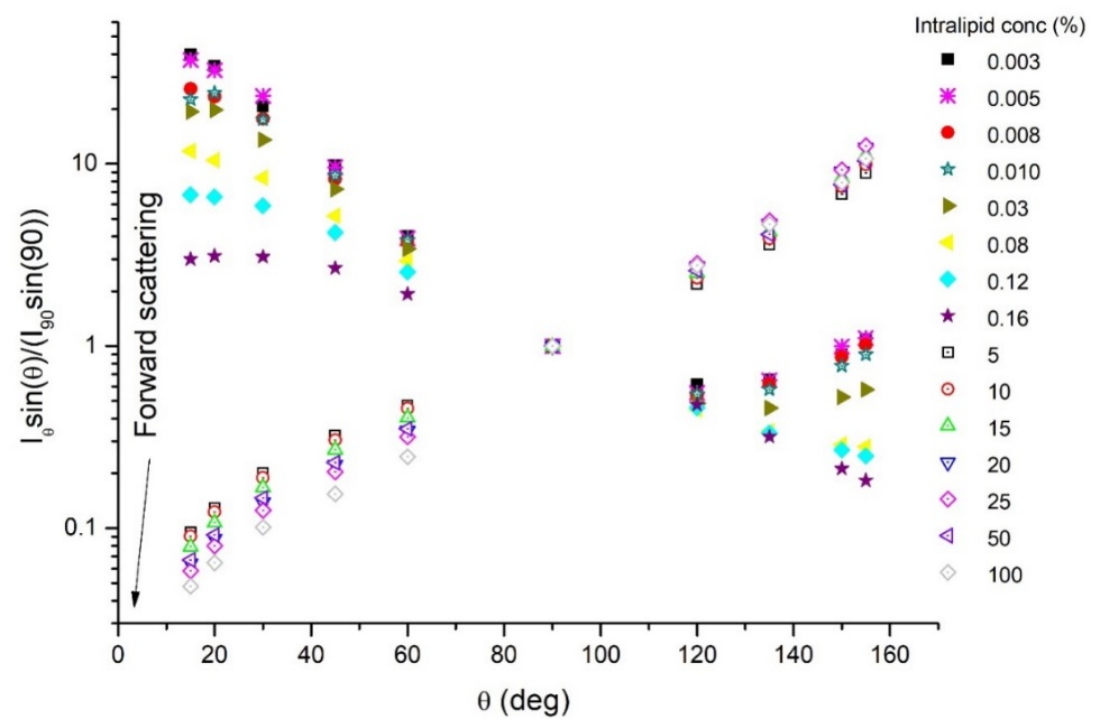

(a)

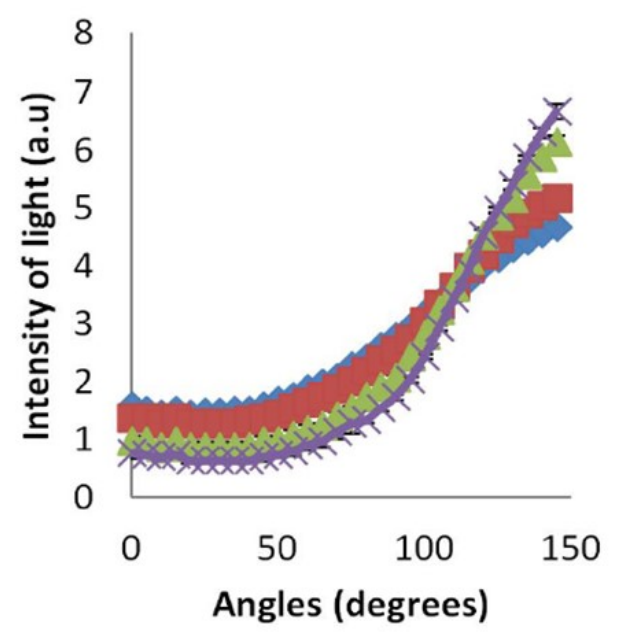

(b)

Figure 5. (a) Relative scattered intensity corrected for volume effects (i.e., $\left.I_{\theta} \sin \theta /\left(I_{90} \sin \left(90^{\circ}\right)\right)\right)$ as a function of the scattering angle obtained for Intralipid suspensions at different concentrations. Intralipid volume concentrations of the considered suspensions are reported in the legend. At $\theta=0^{\circ}$ the forward scattered light was detected, while at $\theta=180^{\circ}$ the backscattered signal was detected. (b) Light intensity as a function of the scattering angle for Intralipid aqueous suspension at different concentrations (diamond (blue), square (red), triangle (green), and $\mathrm{x}$ (purple) in respect to $0.7 \%, 0.875 \%$, $1.4 \%$, and $2.175 \%$ ). Reprinted from. [46].

The overall analysis of these results led to a strong indication of a change in the scattering process, depending on Intralipid concentration, resulting in a variation in the g-factor, and suggests also that it is possible to follow the increase in multiple scattering effects when the Intralipid concentration increases. In fact, for a single scattering process, the light diffusing in backward direction cannot overcome the amount of light travelling in the forward one.

\subsection{DLS Results}

The autocorrelation functions obtained for Intralipid solutions at different volume concentrations are shown in Figure 6a. The shape of the functions was similar for the low scattering samples (0.003-0.16\% Intralipid concentration), while it was completely changed for the highest concentration 
suspension. The correlation functions of the low-scattering samples well resembled the autocorrelation functions typically arising from single scattering samples with freely diffusing scatters (Brownian particles). On the contrary, the function evaluated for the high-scattering sample showed a different time dependence and the intensity signal was correlated over a time shorter than those characterizing the low-scattering samples. The correlation functions obtained for the suspensions with concentrations in the range of $2-10 \%$ show intermediate features. In addition, the characteristic time of the autocorrelation decreased as a function of the Intralipid concentration. These experimental findings confirm that the above-mentioned conditions (single scattering/free diffusion) did not hold for the high-scattering sample. The same results were obtained for Intralipid suspensions at higher concentrations (data not shown).

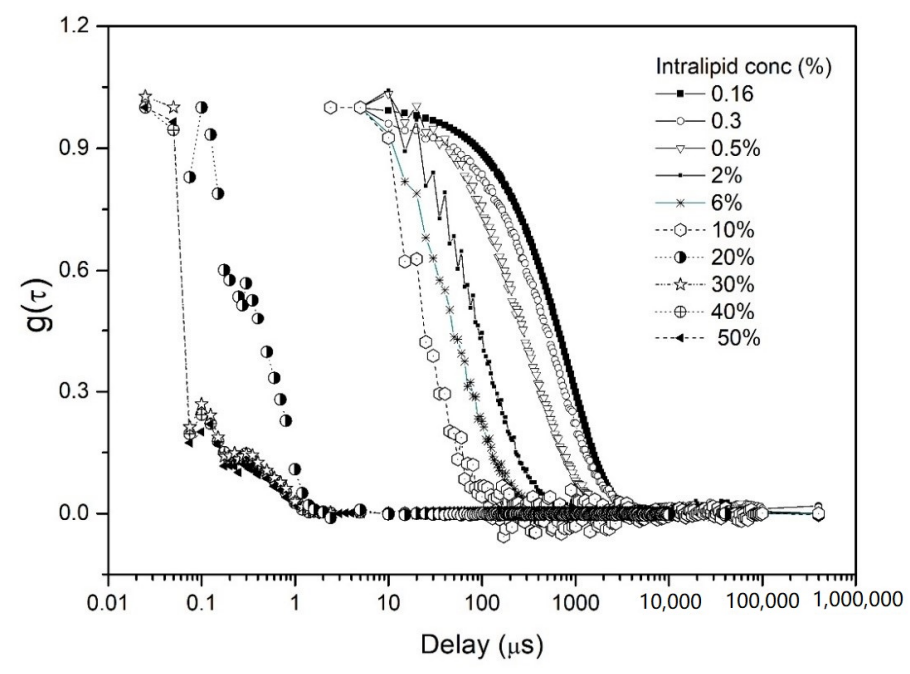

(a)

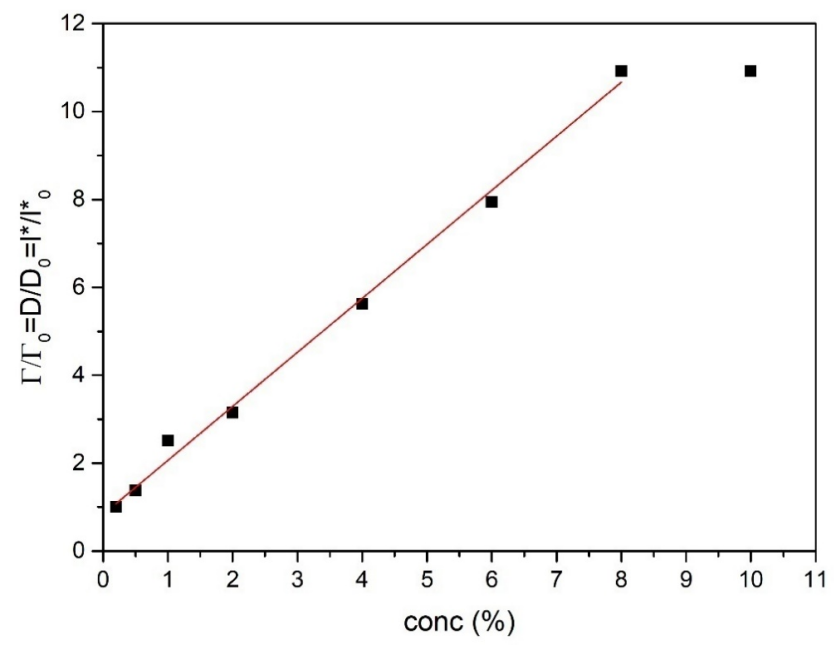

(b)

Figure 6. (a) Normalized autocorrelation functions as obtained by dynamic light scattering (DLS) apparatus in right angle $\left(\theta=90^{\circ}\right)$ collection geometry for suspensions at various volume concentrations of Intralipid. (b) Relative characteristic time (i.e., $\Gamma / \Gamma_{0}$, where the subscript " 0 " indicates that the parameter is the one obtained for the first concentration reported in the figure that was used as reference) vs. Intralipid concentration is reported for the intermediate concentrations (see text). $\Gamma$ and $D$ values were obtained by analyzing the autocorrelation functions, also according to Equations (26) and (27). The red line is the line resulting by performing a linear fit on all the data but the highest concentration. Results of the fitting procedure: intercept $=0.83 \pm 0.16$; slope $=1.23 \pm 0.04$; Pearson's linear correlation coefficient $r=0.998$. 
For the solutions with intermediate concentrations, the characteristic time ( $\Gamma$ see Equation $(26))$ was obtained by analyzing the autocorrelation functions. The resulting values are reported in Figure $6 \mathrm{~b}$, where the value of the relative characteristic time of the particles (i.e., $\Gamma / \Gamma_{0}$, where the subscript " 0 " indicates that the parameter is the one obtained for the lowest concentration reported in the figure that was used as reference) and the corresponding diffusion coefficients are shown, according to Equations (26) and (27).

This suggests that some scattering information about turbid media can be obtained by using DLS even if it cannot be employed for the sizing of the sample components. In fact, it is worth noting that the linear behavior of the $\Gamma / \Gamma_{0}$, as a function of the Intralipid concentration recalls the dependence observed for the reduced scattering coefficient as a function of Intralipid concentration [47].

\subsection{TRT Results}

\subsubsection{Homogeneous Slab Results}

The experimental values obtained for the optical properties of the homogeneous slab samples by using TRT are shown in Figure 7. In particular, the results for the Intralipid suspensions with different scattering properties are shown in Figure 7a. The $\mu_{s}$ ' shows a linear dependence on Intralipid concentration, thus confirming the goodness of Intralipid for obtaining samples with controlled scattering properties in a wide range of $\mu_{s}{ }^{\prime}$ values and the ability of TRT in obtaining the optical properties. The results for the Intralipid suspensions with different absorption properties are shown in Figure $7 \mathrm{~b}$. The measured $\mu_{a}$ of the suspensions shows a linear dependence on ink concentration $\left(\mathrm{C}_{\mathrm{I}}\right)$, thus confirming that Intralipid, in combination with an absorbing agent such as ink, can be used also for obtaining samples with controlled absorption properties in a wide range of $\mu_{a}$ values.

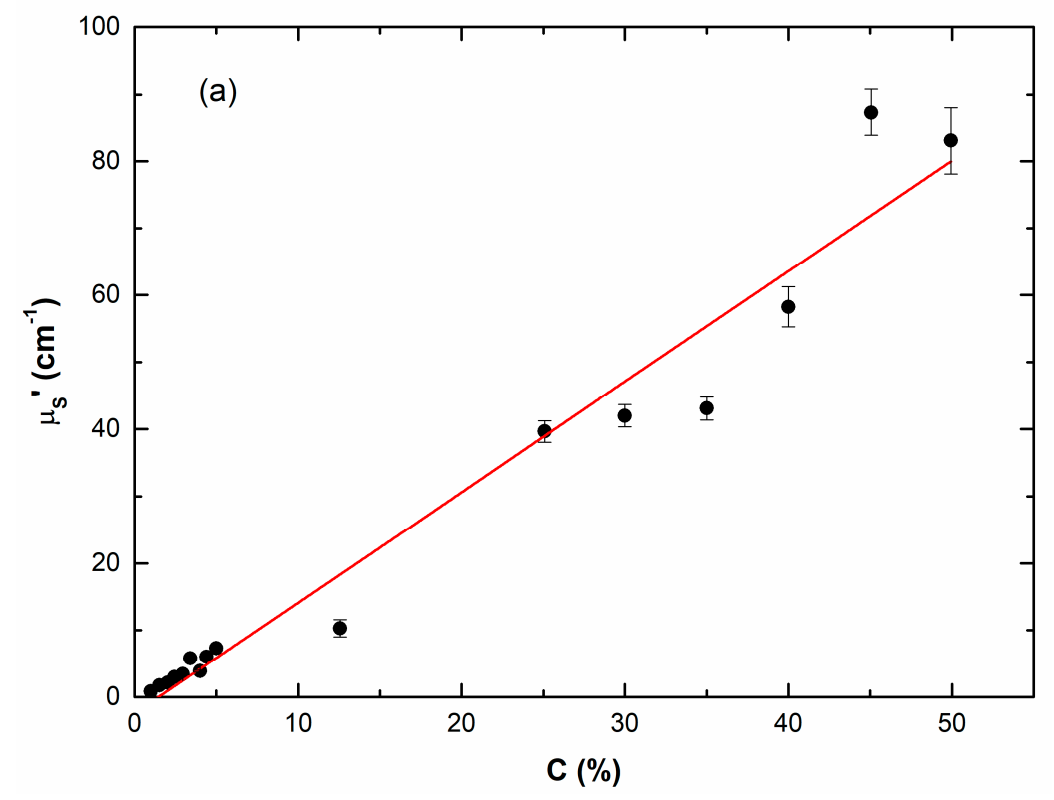

Figure 7. Cont. 


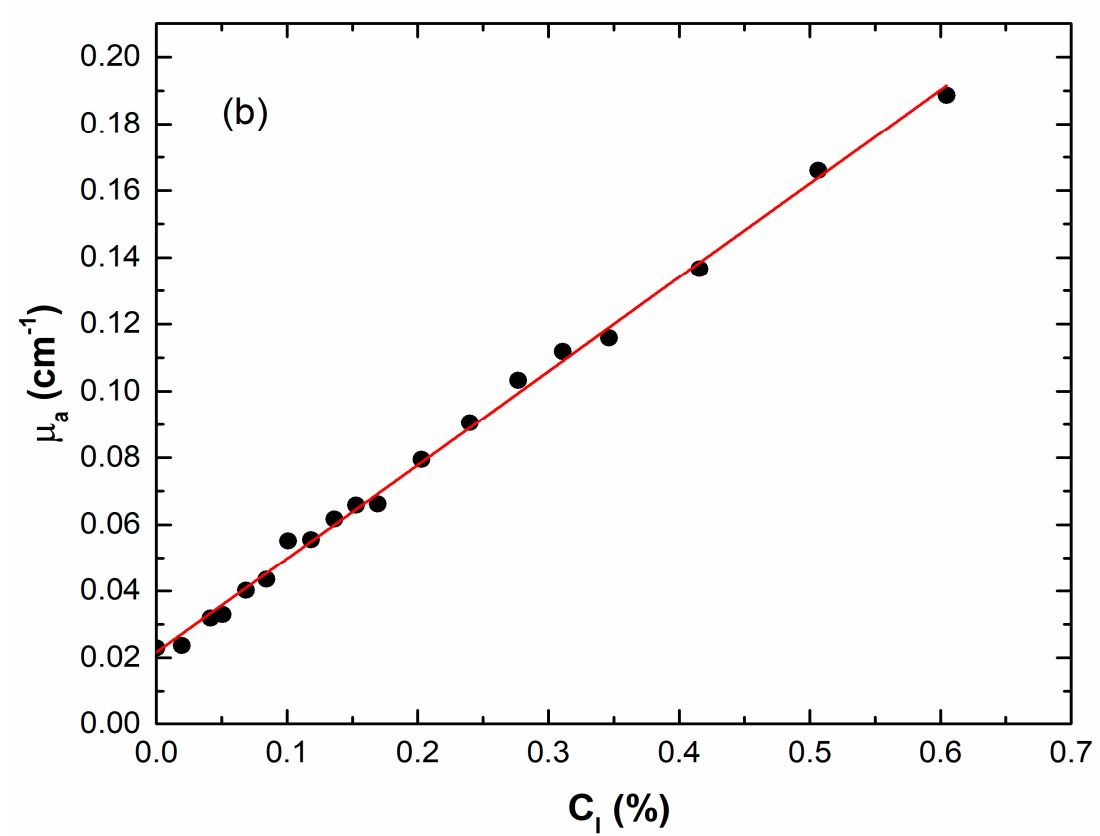

Figure 7. (a) Reduced scattering coefficient of Intralipid suspensions (homogeneous slab) with different Intralipid concentration (C), experimentally obtained by time-resolved transmittance (TRT), as a function of C. Red line: fitted linear curve with intercept $=-0.2 \pm 0.2 \mathrm{~mm}^{-1}$ and slope $=0.165 \% \pm 0.009 \% / \mathrm{mm}^{-1}$; linear correlation coefficient $r=0.98$; absorption coefficient $=0.0035 \pm 0.0008 \mathrm{~mm}^{-1}$. (b) Absorption coefficient of Intralipid suspensions (homogeneous slab) with different ink concentration $\left(\mathrm{C}_{\mathrm{I}}\right.$ ), experimentally obtained by TRT, as a function of $\mathrm{C}_{\mathrm{I}}$. Red line: fitted linear curve with intercept $=0.00216 \pm 0.00001 \mathrm{~mm}^{-1}$ and slope $=0.02804 \% \pm 0.00001 \% / \mathrm{mm}^{-1}$; linear correlation coefficient $\mathrm{r}=1$; reduced scattering coefficient $=0.250 \pm 0.014 \mathrm{~mm}^{-1}$.

\subsubsection{Absorptive Inclusion Results}

According to Section 3.2.2, for this series of measurements, the source beam and the detector were positioned upon the opposite surfaces of the phantom in a coaxial configuration. Each inclusion moved along the x-direction inside the host medium transversally to the source-detector axis. The time-resolved transmittance was measured for each scanning position and the Gaussian perturbation model, which is described in Section 2.2.1 through Equations (15)-(18), was used for retrieving the value of the absorption coefficient of the inclusion $\mu_{a, i n c}$ through a fitting procedure with the experimental measurements. The quantity $\mu_{a, i n c}$ was the only parameter of the fitting because the absorption and the reduced scattering coefficients of the host medium were previously retrieved by fitting the model Equation (14) with the time-resolved transmittance of the phantom without inclusion. Black points of Figure 8 shows the retrieved values of the absorption coefficient $\mu_{a, i n c}$ as function of the scanning position, for all the considered inclusions. As can be seen, a maximum at the location of the center of the inclusion was observed and it was identified as the absorption coefficient value of the inclusion. Experimental points were very well fitted with a Gaussian curve (red line) that allows for a better estimate of the center of the inclusion, its size R, and its absorption coefficient according to Equation (15). The absorption coefficient of the inclusions was retrieved with an error that increased as its value increased. Overall, we obtained an accuracy better than $14 \%$ when the relative perturbation parameter $\Delta \mu_{a} / \mu_{a}$ was less than $140 \%$. 


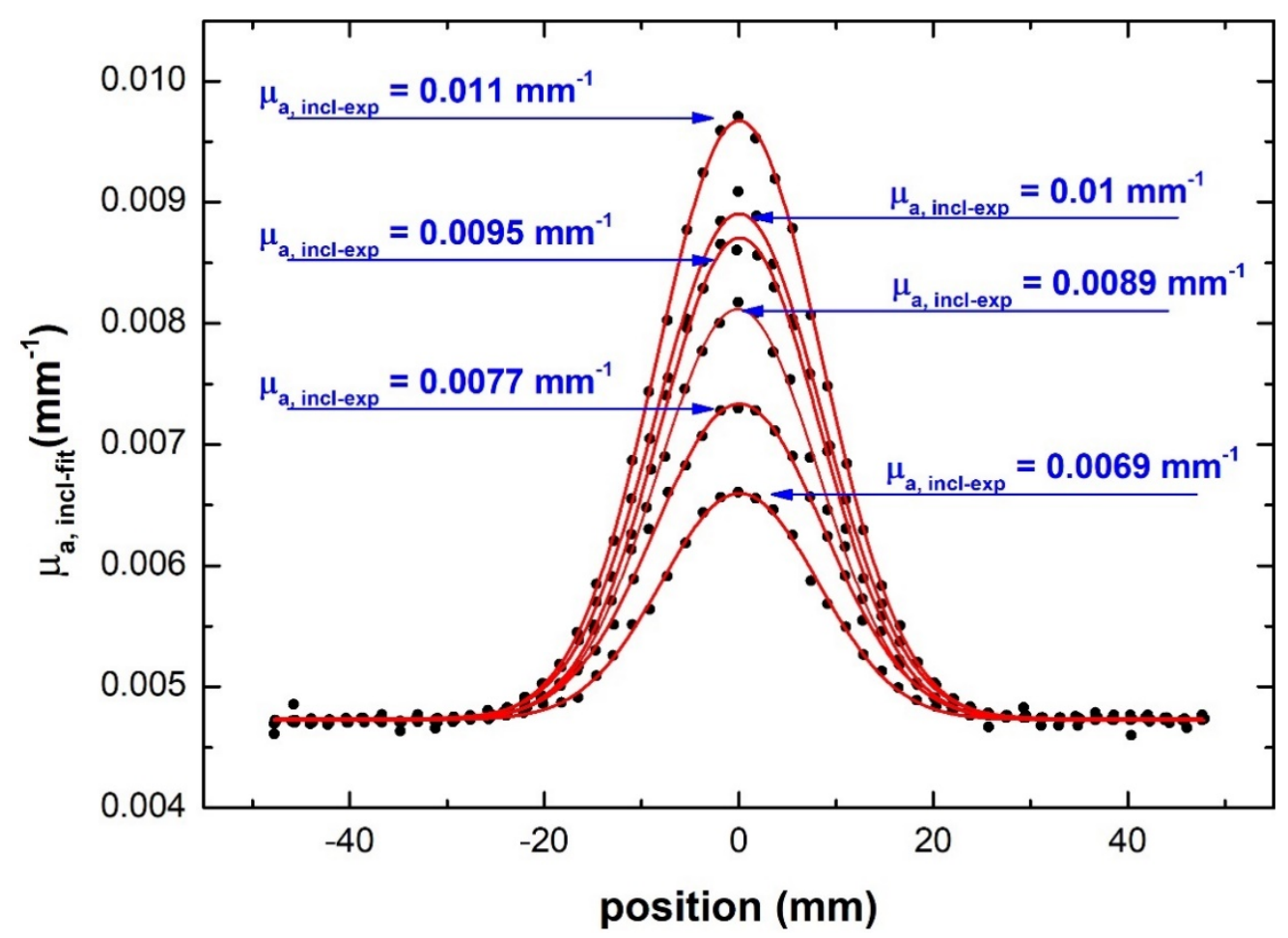

Figure 8. The value of the absorption coefficient estimated at each position as a function of the scansion position for each investigated inclusion.

\section{Conclusions}

Light scattering in turbid media is an attractive field both from a fundamental and an applicative point of view. In the present paper, we showed that the use of SLS, DLS, and TRT techniques allows a detailed characterization of optical properties of turbid media in a large number of cases of interest for many applicative fields such as the food industry, and environmental monitoring and new imaging modalities. Our results also indicate that Intralipid-based samples can be successfully used in a very large range of concentrations for investigating very different experimental conditions, not only the ones related to the development of diagnostic imaging systems, as reported to date. In particular, SLS and DLS results evidence the possibility of using these techniques for characterizing samples in "single scattering" and "multiple scattering" regimes. The use of additional data analysis procedures would increase the amount of extracted information. TRT results show that this technique can be applied to a wide range of experimental situations for evaluating absorption and scattering coefficients and related quantities. It can be also interesting to note that the combined use of more than one technique can give new insights into the optical properties of scattering media. The SLS apparatus and TRT experimental set-ups can be used for analyzing the elastic scattering processes from different points of view. For example, SLS and TRT give static and dynamic information, respectively. In this way, a great knowledge of the relationship between static and dynamic scattering properties can be obtained. In fact, the main results of SLS experiments concern static scattering properties (for example, the transport mean free path), while the outcomes of TRT experiments are dynamic scattering parameters (for example, the diffusion coefficient). Researchers can better understand effects such as coherent backscattering, weak localization, short- and long-range spatial correlation of light fluctuations, and speed of light in random media when very peculiar materials are considered for the experimental study of the relations between static and dynamic parameters. Moreover, the use of inclusions allowed us to theoretically and experimentally investigate the effects of local changes in bulk optical properties on light scattering. First order approximation contrast functions are widely used to predict the light scattering behavior in the presence of local inhomogeneities in an otherwise 
homogeneous bulk. The limited accuracy of this method has led to iterative schemes that improve the accuracy of the calculated diffuse intensity. The closed-form solution to the steady-state diffusion equation in the presence of inhomogeneities described here has the advantage of being amenable both to weak and strong optical absorbing defects by using a simple analytical expression. The related results obtained here are of fundamental importance since they denote the possibility to go beyond the limits of perturbative approaches to diffusion approximation theories in turbid media.

Author Contributions: Conceptualization, M.L. and I.D.; methodology, M.L., I.D., R.E.; software, I.D., R.E.; investigation, I.D., R.E.; data curation, I.D., R.E.; writing-original draft preparation, M.L.; writing-review and editing, M.L., I.D., R.E. All authors have read and agreed to the published version of the manuscript.

Funding: This research received no external funding.

Conflicts of Interest: The authors declare no conflict of interest.

\section{References}

1. Ruiz, C.C.; Molina-Bolıvar, J.A.; Aguiar, J. Thermodynamic and Structural Studies of Triton X-100 Micelles in Ethylene Glycol-Water Mixed Solvents. Langmuir 2001, 17, 6831-6840. [CrossRef]

2. Berne, B.J.; Pecora, R. Dynamic Light Scattering; Wiley-Interscience: New York, NY, USA, 1976.

3. Kerker, M. The Scattering of Light, and Other Electromagnetic Radiations; Academic Press: New York, NY, USA, 1969.

4. Pine, D.J.; Weitz, D.A.; Chaikin, P.M.; Herbolzheimer, E. Diffusing-wave spectroscopy. Phys. Rev. Lett. 1988, 60, 1134-1137. [CrossRef] [PubMed]

5. Corredig, M.; Alexander, M. Food emulsions studied by DWS: Recent advances. Trends Food Sci. Technol. 2008, 19, 67-75. [CrossRef]

6. Kwan, T.O.C.; Reis, R.; Siligardi, G.; Hussain, R.; Cheruvara, H.; Moraes, I. Selection of Biophysical Methods for Characterisation of Membrane Proteins. Int. J. Mol. Sci. 2019, 20, 2605. [CrossRef] [PubMed]

7. Badruddoza, A.Z.M.; MacWilliams, S.V.; Sebbenb, D.A.; Krasowska, M.; Beattie, D.; Durian, D.J.; Ferri, J.K. Diffusing wave spectroscopy (DWS) methods applied to double emulsions. Curr. Opin. Colloid Interface Sci. 2018, 37, 74-87. [CrossRef]

8. Delfino, I.; Piccolo, C.; Lepore, M. Experimental study of short- and long-time diffusion regimes of spherical particles in carboxymethylcellulose solutions. Eur. Pol. J. 2005, 41, 1772-1780. [CrossRef]

9. Martins, S.; Martins, I.C.; Santos, N.C. Methods for Lipid Droplet Biophysical Characterization in Flaviviridae Infections. Front. Microbiol. 2018, 9, 1951. [CrossRef]

10. Jain, K.; Thareja, S. In vitro and in vivo characterization of pharmaceutical nanocarriers used for drug delivery. Artif. Cells Nanomed. Biotechnol. 2019, 47, 524-539. [CrossRef]

11. Niu, F.; Ahmada, M.; Fana, J.; Ritzoulis, C.; Chen, J.; Luo, Z.; Pana, W. The application of diffusing wave spectroscopy (DWS) in soft foods. Food Hydrocoll. 2019, 96, 671-680. [CrossRef]

12. Xu, J.; Liu, S.X.; Boddu, V.M. Micro-rheological and micro-heterogeneity properties of soluble glutinous rice starch (SGRS) solutions studied by diffusing wave spectroscopy (DWS). J. Food Meas. Charact. 2019, 13, 2822-2827. [CrossRef]

13. Carvalho, P.M.; Felício, M.R.; Santos, N.C.; Gonçalves, S.; Domingues, M.M. Application of Light Scattering Techniques to Nanoparticle Characterization and Development. Front. Chem. 2018, 6, 237. [CrossRef] [PubMed]

14. Maguire, C.M.; Rösslein, M.; Wickc, P.; Prina-Mello, A. Characterisation of particles in solution-a perspective on light scattering and comparative technologies. Sci. Technol. Adv. Mater. 2018, 19, 732-745. [CrossRef] [PubMed]

15. Sharma, S.; Jaiswal, S.; Duffy, B.; Jaiswal, A.K. Nanostructured Materials for Food Applications: Spectroscopy, Microscopy and Physical Properties. Bioengineering 2019, 6, 26. [CrossRef] [PubMed]

16. Minton, A.P. Recent applications of light scattering measurement in the biological and biopharmaceutical sciences. Anal. Biochem. 2016, 501, 4-22. [CrossRef]

17. Zhou, W.; Kholiqov, O.; Chong, S.P.; Srinivasan, V.J. Highly parallel, interferometric diffusing wave spectroscopy for monitoring cerebral blood flow dynamics. Optica 2018, 5, 518-527. [CrossRef] [PubMed] 
18. Yamada, Y.; Suzuki, H.; Yamashita, Y. Time-Domain Near-Infrared Spectroscopy and Imaging: A Review. Appl. Sci. 2019, 9, 1127. [CrossRef]

19. Delfino, I.; Lepore, M.; Indovina, P.L. Experimental tests of different solutions to the diffusion equation for optical characterization of scattering media by time-resolved transmittance. Appl. Opt. 1999, 38, 4228-4236. [CrossRef]

20. Esposito, R.; Brambilla, M.; Pifferi, A.; Spinelli, L.; De Nicola, S.; Lepore, M. Depth dependence of estimated optical properties of a scattering inclusion by time-resolved contrast function. Opt. Express 2008, 16, 17667-17681. [CrossRef]

21. Kholiqov, O.; Zhou, W.; Zhang, T.; Le, V.N.D.; Srinivasan, V.J. Time-of-flight resolved light field fluctuations reveal deep human tissue physiology. Nat. Commun. 2020, 11, 391. [CrossRef]

22. Nicolai, B.M.; Beullens, K.; Bobelyn, E.; Peirs, A.; Saeys, W.; Theron, K.I.; Lammertyn, J. Review Nondestructive measurement of fruit and vegetable quality by means of NIR spectroscopy: A review. Postharvest Biol. Technol. 2007, 46, 99-118. [CrossRef]

23. Torricelli, A.; Spinelli, L.; Contini, D.; Vanoli, M.; Rizzolo, A.; Zerbini, P.E. Time-resolved reflectance spectroscopy for non-destructive assessment of food quality. Sens. Instrum. Food Qual. Saf. 2008, 2, 82-89. [CrossRef]

24. Torricelli, A.; Contini, D.; Mora, A.D.; Martinenghi, E.; Tamborini, D.; Villa, F.; Tosi, A.; Spinelli, L. Recent advances in time-resolved nir spectroscopy for nondestructive assessment of fruit quality. Chem. Eng. Trans. 2015, 44, 43-48.

25. Rizzolo, A.; Vanoli, M. Time-Resolved Technique for Measuring Optical Properties and Quality of Food. In Light Scattering Technology for Food Property, Quality and Safety Assessment; Lu, R., Ed.; CRC Press Taylor \& Francis Group: Oxfordshire, UK, 2016; pp. 187-224. [CrossRef]

26. Milej, D.; Kruczkowski, M.; Kacprzak, M.; Sawosz, P.; Maniewski, R.; Liebert, A. Estimation of light detection efficiency for different light guides used in time-resolved near-infrared spectroscopy. Biocybern. Biomed. Eng. 2015, 35, 227-231. [CrossRef]

27. Park, J.H.; Park, J.; Lee, K.R.; Park, Y.K. Disordered optics: Exploiting multiple light scattering and wavefront shaping for nonconventional optical elements. Adv. Mater. 2019, 32, 1903457. [CrossRef]

28. Bohren, C.F.; Huffman, D.R. Absorption and Scattering of Light by Small Particles; John Wiley \& Sons: New York, NY, USA, 1983.

29. Ishimaru, A. Wave Propagation and Scattering in Random Media; Academic Press: New York, NY, USA, 1978.

30. Pierrat, R.; Greffet, J.-J.; Carminati, R. Photon diffusion coefficient in scattering and absorbing media. J. Opt. Soc. Am. A 2006, 23, 1106-1110. [CrossRef]

31. Delfino, I.; Esposito, R.; Piccirillo, B.; Lepore, M. Static and dynamic light scattering properties of Intralipid aqueous suspensions for tissue phantoms preparation and calibration. Proc. SPIE 2008, 6870, 68700P. [CrossRef]

32. Cletus, B.; Künnemeyer, R.; Martinsen, P.; McGlone, V.A. Temperature-dependent optical properties of Intralipid measured with frequency-domain photon-migration spectroscopy. J. Biomed. Opt. 2010, 15, 017003. [CrossRef]

33. Ninni, P.D.; Martelli, F.; Zaccanti, G. Intralipid: Towards a diffusive reference standard for optical tissue phantoms. Phys. Med. Biol. 2011, 56, N21-N28. [CrossRef]

34. Rowe, P.I.; Künnemeyer, R.; McGlone, R.; Talele, S.; Martinsen, P.; Oliver, R. Thermal stability of intralipid optical phantoms. Appl. Spectrosc. 2013, 67, 993-996. [CrossRef]

35. Cipelletti, L.; Trappe, V.; Pine, D.J. Scattering Techniques. In Fluids, Colloids and Soft Materials: An Introduction to Soft Matter Physics; Nieves, A.F., Puertas, A.F., Eds.; John Wiley \& Sons, Inc.: New York, NY, USA, 2016.

36. Murphy, R.M. Murphy Static and dynamic light scattering of biological macromolecules: What can we learn? Opin. Biotechnol. 1997, 8, 25-30. [CrossRef]

37. Sassaroli, A.; Martelli, F.; Fantini, S. Perturbation theory for the diffusion equation by use of the moments of the generalized temporal point-spread function. III. Frequency-domain and time-domain results. J. Opt. Soc. Am. A 2010, 27, 1723-1742. [CrossRef] [PubMed]

38. Contini, D.; Martelli, F.; Zaccanti, G. Photon migration through a turbid slab described by a model based on diffusion approximation. I. Theory. Appl. Opt. 1997, 36, 4587-4599. [CrossRef] [PubMed]

39. Esposito, R.; Martelli, F.; De Nicola, S. Closed-form solution of the steady-state photon diffusion equation in the presence of absorbing inclusions. Opt. Lett. 2014, 39, 826-829. [CrossRef] [PubMed] 
40. Van Staveren, H.J.; Moes, C.J.; van Marie, J.; Prahl, S.A.; van Gemert, M.J.C. Light scattering in Intralipid-10\% in the wavelength range of 400-1100nm. Appl. Opt. 1991, 30, 4507-4514. [CrossRef] [PubMed]

41. Cubeddu, R.; Pifferi, A.; Taroni, P.; Torricelli, A.; Valentini, G. A solid tissue phantom for photon migration studies. Phys. Med. Biol. 1997, 42, 1971-1979. [CrossRef] [PubMed]

42. Stetefeld, J.; McKenna, S.A.; Patel, T.R. Dynamic light scattering: A practical guide and applications in biomedical sciences. Biophys. Rev. 2016, 8, 409-427. [CrossRef] [PubMed]

43. Chernomordik, V.; Gandjbakhche, A.; Lepore, M.; Esposito, R.; Delfino, I. Depth dependence of the analytical expression for the width of the point spread function (spatial resolution) in time resolved transillumination. J. Biomed. Opt. 2001, 6, 441-445. [CrossRef]

44. Delfino, M.I.; Lepore, M.; Indovina, P.L. Experimental evaluation of absorption coefficient in scattering media using different solutions to the diffusion equation. Phys. Med. 2002, XVIII, 124-136.

45. Wiersma, D.S.; Muzzi, A.; Colocci, M.; Righini, R. Time-resolved experiments on light diffusion in anisotropic random media. Phys. Rev. E 2000, 62, 6681-6687. [CrossRef]

46. Feder, I.; Duadi, H.; Fixler, D. Experimental system for measuring the full scattering profile of circular phantoms. Biomed. Opt. Express 2015, 6, 2877-2886. [CrossRef]

47. Spinelli, L.; Martelli, F.; Farina, A.; Pifferi, A.; Torricelli, A.; Cubeddu, R.; Zaccanti, G. Calibration of scattering and absorption properties of a liquid diffusive medium at NIR wavelengths. Time-resolved method. Opt. Express 2007, 15, 6589-6604. [CrossRef] [PubMed]

(C) 2020 by the authors. Licensee MDPI, Basel, Switzerland. This article is an open access article distributed under the terms and conditions of the Creative Commons Attribution (CC BY) license (http://creativecommons.org/licenses/by/4.0/). 